\title{
Jahresinhaltsverzeichnis 55. Jahrgang 2007
}

Editorial

\section{ARTIKEL}

Eva Blömeke / Michel Clement / Ilaha Mahmudova / Frank Sambeth

Richard Collins

Beatrice Dernbach / Judith Roth

Thomas Hanitzsch

Thomas Hanitzsch /

Klaus-Dieter Altmeppen

Dorothée Hefner / Christoph

Klimmt / Gregor Daschmann

Jochen Hoffmann

Christoph Klimmt / Petra Netta / Peter Vorderer

Castulus Kolo / Robin Meyer-Lucht

Michael Meyen

Irene Neverla /

Hanns-Christian Kamp /

Mascha Brichta / Dieter K. Lüdecke
Status quo der betriebswirtschaftlichen Erfolgsfaktorenforschung bei Büchern. Eine kritische Analyse der empirischen Literatur . . . . . . . . . . . 3/412

The BBC and „public value“............. 2/164

Literalität des Alltags: Von Scannern, Gehern und Direkteinsteigern - Eine Typologie von Verhaltensmustern beim Zeitunglesen . . . . . . . . . . . . 1/24

Journalismuskultur: Zur Dimensionierung eines zentralen Konstrukts der kulturvergleichenden Journalismusforschung ................ 2/185

Über das Vergleichen: Komparative Forschung in deutschen kommunikationswissenschaftlichen Fachzeitschriften 1948-2005 ................ 2/185

Typisch Türke? Die Folgen der Nationalitätsnennung für die Bewertung von Akteuren in der Nachrichtenrezeption .................... 4/575

Mitgliederpresse: Journalismus für die Organisation, PR für die Gesellschaft . . . . . . . . . . . . 4 4/555

Entertainisierung der Wahlkampfkommunikation. Der Einfluss von Humor auf die Wirkung negativer Wahlwerbung ..................... 3/390

Erosion der Intensivleserschaft. Eine Zeitreihenanalyse zum Konkurrenzverhältnis von Tageszeitungen und Nachrichtensites ............ 4/513

Medienwissen und Medienmenüs als kulturelles Kapital und als Distinktionsmerkmale. Eine Typologie der Mediennutzer in Deutschland ........... 3/333

Zum Informationshandeln von Patienten und zur Rolle des Internets im Krankheitsfall - Empirische Befunde aus einer Befragung von Akromegalie-Patienten ...................... $1 / 43$ 
Christian Pentzold /

Sebastian Seidenglanz / Claudia Fraas / Wikis - Bestandsaufnahme eines Forschungsfeldes Peter Ohler und Skizzierung eines integrativen Analyserahmens 1/61

Bertram Scheufele / Carolin Gasteiger Berichterstattung, Emotionen und politische Legitimierung. Eine experimentelle Untersuchung zum Einfluss der Politikberichterstattung auf die Legitimierung politischer Entscheidungen am Beispiel von Bundeswehreinsätzen ...............4 4/534

Birgit Stark

Programmauswahl in digitalen Fernsehwelten: Der EPG als Gatekeeper? Ergebnisse einer Nutzerbefragung.

Rudolf Stöber

Vom deutsch-französischen Krieg zum Zweiten Weltkrieg. Kriegsberichterstattung zwischen journalistischen Routinen und politischer Propaganda ................................. 2/204

Regina Vetters

Vor Ort in Europa. Ein Vergleich der EU-Berichterstattung deutscher Qualitäts- und Regionalzeitungen ............................. 3/355

\section{LITERATUR}

Reihe „Klassiker der Kommunikations- und Medienwissenschaft heute“

Thymian Bussemer

Jürgen Wilke

Carsten Winter

\section{Besprechungen}

Silke Adam

Stefanie Averbeck
Paul Felix Lazarsfeld und die Etablierung der Kommunikationsforschung als empirische Sozialwissenschaft ......................... $1 / 80$

Nicht nur ein Theoretiker der Öffentlichen Meinung: Walter Lippmann Revisited ........... 4/595

Raymond Williams (1921-1988) - Medien- und Kommunikationsforschung für die Demokratisierung von Kultur und Gesellschaft ........... 2/247

Markus Lehmkuhl: Massenmedien und interpersonale Kommunikation. Eine explorative Studie am Beispiel BSE. Konstanz: UVK, 2006 . . . . . . . 2/265

Sabine Seggelke: Frankreichs Staatspräsident in der politischen Kommunikation. Öffentlichkeitsarbeit in der V. Republik. Münster: LIT, 2007 . . . . . . . 4/613 
Christian Beck

Helena Bilandzic

Joan Kristin Bleicher

Thymian Bussemer

Mark D. Cole

Christoph Degenhart

Nicola Döring

Katrin Döveling

Indira Dupuis

Wolfgang Eichhorn

Susanne Fengler

Rüdiger Funiok
Andreas Schelske: Soziologie vernetzter Medien. Grundlagen computervermittelter Vergesellschaftung. München: Oldenbourg, 2007 . . . . . . . . . 4 4/615

Ruth Ayaß / Jörg Bergmann (Hrsg.): Qualitative Methoden der Medienforschung, Reinbek: Rowohlt, $2006 \ldots \ldots \ldots \ldots \ldots \ldots \ldots \ldots \ldots . \ldots . \ldots .442$

Yasmin Hoffmann / Walburga Hülk / Volker Roloff (Hrsg.): Alte Mythen - Neue Medien. Heidelberg: Univ.-Verl. Winter, 2006

Magnus-Sebastian Kutz: Public Relations oder Propaganda? Die Öffentlichkeitsarbeit der US-Administration zum Krieg gegen den Irak 2003. Münster u. a.: LIT, 2006

Uwe Jürgens: Marktzutrittsregulierung elektronischer Informations- und Kommunikationsdienste. Baden-Baden: Nomos, 2005 . . . . . . . . . . . . . 4/616

Stephanie Reese: Der Funktionsauftrag des öffentlich-rechtlichen Rundfunks vor dem Hintergrund der Digitalisierung. Frankfurt a. M.: Peter Lang, $2006 \ldots \ldots \ldots \ldots \ldots \ldots \ldots \ldots$. . . . . . . . . . . 3/445

Gerhard Banse (Hrsg.): Neue Kultur(en) durch neue Medien (?) Das Beispiel Internet. Berlin: Trafo, 2005

William Uricchio / Susanne Kinnebrock (Hrsg.): Media Cultures. Heidelberg: Universitätsverlag Winter, 2006 . . . . . . . . . . . . . . . . . . . . . . . . . 1/102

Katrin Voltmer (Hrsg.): Mass Media and Political Communication in New Democracies. London: Routledge, $2006 \ldots \ldots \ldots \ldots \ldots \ldots \ldots \ldots \ldots$. 3/447

Bernd Klammer: Empirische Sozialforschung. Eine Einführung für Kommunikationswissenschaftler und Journalisten. Konstanz: UVK, 2005 . . . . . . 3/448

Sandra Hermes: Qualitätsmanagement in Nachrichtenredaktionen. Köln: von Halem, 2006 . . . . . . . 1/103

Birgitta Derenthal. Medienverantwortung in christlicher Perspektive: Ein Beitrag zu einer praktischtheologischen Medienethik: Münster/Hamburg/ London: LIT, $2006 \ldots \ldots \ldots \ldots \ldots \ldots \ldots \ldots \ldots$. . . . . . . . . . . 
Rüdiger Funiok

Udo Göttlich

Thomas Hanitzsch

Jürgen Heinrich

Andreas Hepp

Heinz Hiebler

Christoph Hilgert

Thomas Hoeren

Wolfgang Hofkirchner

Bastian Jenderek

Heike Klippel

Steffen Kolb
Katrin Döveling: Emotionen - Medien - Gemeinschaft. Eine kommunikationssoziologische Analyse.

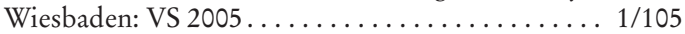

Stephan A. Weichert: Die Krise als Medienereignis. Über den 11. September im Deutschen Fernsehen. Köln: von Halem, 2006 . . . . . . . . . . . . . . . . 4 4/618

Margreth Lünenborg: Journalismus als kultureller Prozess. Zur Bedeutung von Journalismus in der Mediengesellschaft - ein Entwurf. Wiesbaden: VS, 2005 . . . . . . . . . . . . . . . . . . . . . . . . . . 3/450

Kai Wengenroth: Neue Erlösformen im deutschen Fernsehen. Entwicklung und Zukunft der Fernsehfinanzierung. Saarbrücken: VDM, 2006....... 3/452

Michael Jäckel (Hrsg.): Mediensoziologie: Grundfragen und Forschungsfelder. Wiesbaden: VS, 2005 2/270

Wolfgang Raible: Medien-Kulturgeschichte. Mediatisierung als Grundlage unserer kulturellen Entwicklung. Heidelberg: Univ.-Verl. Winter, 2006 . . 3/453

Clas Dammann: Stimme aus dem Äther - Fenster zur Welt. Die Anfänge von Radio und Fernsehen in Deutschland. Köln/Weimar/Wien: Böhlau, 2005 . . 2/271

Gabriele Siegert / Rolf H. Weber / Frank Lobigs / Dirk Spacek: Der Schutz innovativer publizistischer Konzepte im Medienwettbewerb. Eine medienökonomische und medienrechtliche Untersuchung. Baden-Baden: Nomos, 2006 . . . . . . . . . . . . . . . . 3/454

Andreas Hepp / Friedrich Krotz / Shaun Moores / Carsten Winter (Hrsg.): Konnektivität, Netzwerke und Fluss. Konzepte gegenwärtiger Medien-, Kommunikations- und Kulturtheorie. Wiesbaden: VS, $2006 \ldots \ldots \ldots \ldots \ldots \ldots \ldots \ldots \ldots$. . . . . . . . . . . . . .

Holger Schramm / Werner Wirth / Helena Bilandzic (Hrsg.): Empirische Unterhaltungsforschung. Studien zu Rezeption und Wirkung von medialer Unterhaltung. München: R. Fischer, 2006 . . . . . . 2/273

Jörg Schweinitz: Film und Stereotyp - eine Herausforderung für das Kino und die Filmtheorie. Zur Geschichte eines Mediendiskurses. Berlin: Akademie Verl., 2006 ..................... 1/106

Thomas Wägenbaur (Hrsg.): Medienanalyse. Methoden, Ergebnisse, Grenzen. Baden-Baden: Nomos, 2007 ........................ 4/621 
Friedrich Krotz

Hans-Dieter Kübler

Tilman Lang

Mirko Marr

Marcus Maurer

Torsten Maurer

Klaus Merten

Lothar Mikos

Wiebke Möhring

Christoph Neuberger

Ingrid Paus-Hasebrink

Leonard Reinecke
Natascha Gentz / Stefan Kramer (Eds.): Globalization, Cultural Identities, and Media Representations. Albany New York: State University of New York Press, 2006 . . . . . . . . . . . . . . . . . 2/276

Gudrun Marci-Boehncke / Matthias Rath (Hrsg.): Jugend - Werte -Medien. Der Diskurs. Weinheim: Beltz, 2006......................... 4/622

Mike Sandbothe / Ludwig Nagl (Hrsg.): Systematische Medienphilosophie. Berlin: Akademie, 2005. . 4/624

Nicole Zillien: Digitale Ungleichheit. Neue Technologien und alte Ungleichheiten in der Informationsund Wissensgesellschaft. Wiesbaden: VS, 2006 ... 4/627

Tanjev Schultz: Geschwätz oder Diskurs? Die Rationalität politischer Talkshows im Fernsehen. Köln:

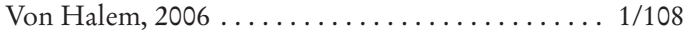

Hanko Bommert / Andrea Voß-Frick: Fakten und Images. Interviews im dualen System des deutschen Fernsehens. Münster: LIT, 2005 . . . . . . . . . . . 2/277

Volker Gehrau / Benjamin Fretwurst / Birgit Krause / Gregor Daschmann (Hrsg.): Auswahlverfahren in der Kommunikationswissenschaft. Köln: Von Halem, $2005 \ldots \ldots \ldots \ldots \ldots \ldots \ldots \ldots$. . . . . . . . . . . . . . .

Ingrid Paus-Hasebrink / Jens Woelke / Michelle Bichler / Alois Pluschkowitz: Einführung in die Audiovisuelle Kommunikation. München: Oldenbourg, $2006 \ldots \ldots \ldots \ldots \ldots \ldots \ldots \ldots \ldots \ldots \ldots$. . . . . . . . .

Urs Dahinden / Sabina Sturzenegger / Alessa C. Neuroni: Wissenschaftliches Arbeiten in der Kommunikationswissenschaft. Bern: Haupt, 2006 . . . 3 3/456

Johannes Raabe: Die Beobachtung journalistischer Akteure. Optionen einer empirisch-kritischen Journalismusforschung. Wiesbaden: VS, 2005 . . . . . 3/457

Werner Wirth / Hans-Jörg Stiehler / Carsten Wünsch (Hrsg.): Dynamisch-transaktional denken. Theorie und Empirie der Kommunikationswissenschaft. Für Werner Früh. Köln: von Halem, 2007 . . . . . . . . 4 4/628

Sabine Geyer: Computerspiele, Gewalt und Terror Management. Grundlagen, Theorie, Praxis. Saarbrücken: VDM, $2006 \ldots \ldots \ldots \ldots \ldots \ldots \ldots$. 4/631 
Gabi Reinmann

Jutta Röser

Armin Rott

Georg Ruhrmann

Helmut Schanze

Bertram Scheufele

Ulrich Schmitz

Armin Scholl

Holger Schramm

Hans-Jörg Stiehler

Joachim Trebbe

Jeffrey Wimmer
Caja Thimm (Hrsg.): Netz-Bildung. Lehren und Lernen mit neuen Medien in Wissenschaft und Wirtschaft. Berlin: Lang, 2005 ................. 1/110

Thomas Berker / Maren Hartmann / Yves Punie / Katie J. Ward (Eds.): Domestication of Media and Technology. Maidenhead: Open University Press,

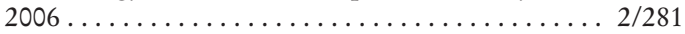

Robert G. Picard (Hrsg.): Media Product Portfolios. Issues in Management of Multiple Products and Services. Mahway: Lawrence Erlbaum Associates, 2005

Andreas Ziemann (Hrsg.): Medien der GesellschaftGesellschaft der Medien. Konstanz: UVK, 2006. . . 3/458

Claudia Dittmar / Susanne Vollberg (Hrsg.): Alternativen im DDR-Fernsehen? Die Programmentwicklung 1981 bis 1985. Leipzig: Universitätsverlag

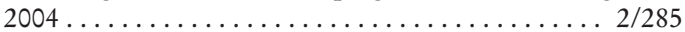

Martina Leonarz: Gentechnik im Fernsehen. Konstanz: UVK, $2006 \ldots \ldots \ldots \ldots \ldots \ldots \ldots \ldots \ldots$. 2/286

Wilhelm Hofmann (Hrsg.): Bildpolitik - Sprachpolitik. Untersuchungen zur politischen Kommunikation in der entwickelten Demokratie. Münster: LIT,

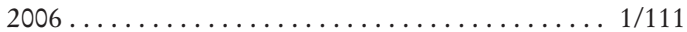

Klaus Forster: Journalismus im Spannungsfeld zwischen Freiheit und Verantwortung. Das Konzept des „Public Journalism“ und seine empirische Relevanz. Köln: von Halem, 2006 . . . . . . . . . . . . . . . 3/460

Steven Brown / Ulrik Volgsten (Hrsg.): Music and Manipulation. On the Social Uses and Social Control of Music. New York, Oxford: Berghahn Books,

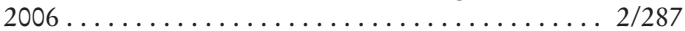

Christian Schütte: Matchwinner und Pechvögel. Ergebniserklärung in der Fußballberichterstattung in Hörfunk, Internet, Fernsehen und Printmedien. Münster: LIT, 2006................... 4/632

Marcus Maurer / Carsten Reinemann: Medieninhalte. Eine Einführung. Wiesbaden: VS, 2006 ..... 1/113

Urs Dahinden: Framing. Eine integrative Theorie der Massenkommunikation. Konstanz: UVK, 2006 
Carsten Winter

Jens Wolling

Hans J. Wulff

Nicole Zillien

Oliver Zöllner

Zeitschriftenlese

Literaturverzeichnis

English abstracts
Heinz Bonfadelli / Otfried Jarren / Gabriele Siegert (Hrsg.): Einführung in die Publizistikwissenschaft. 2., vollständig überarbeitete Aufl. Bern: Haupt Ver-

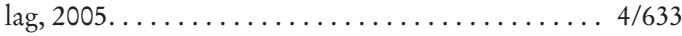

Torsten Maurer: Fernsehnachrichten und Nachrichtenqualität. Eine Längsschnittstudie zur Nachrichtenentwicklung in Deutschland. München: R.

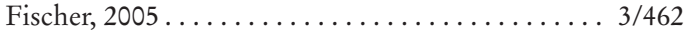

Frank Bösch / Manuel Borutta (Hrsg.): Die Massen bewegen. Medien und Emotionen in der Moderne. Frankfurt/New York: Campus, 2006. . . . . . . . . 3/464

Andreas Ziemann: Soziologie der Medien. Bielefeld: transcript, $2006 \ldots \ldots \ldots \ldots \ldots \ldots \ldots \ldots \ldots \ldots \ldots \ldots$ 1/114

Pamela J. Shoemaker / Akiba Cohen: News Around the World. Content, Practitioners, and the Public. New York/London: Routledge, 2006. ......... 4/637 